\title{
Plasma Concentrations of Neuroactive Steroids before and after Repetitive Transcranial Magnetic Stimulation (rTMS) in Major Depression
}

Frank Padberg, M.D., Flavia di Michele, M.D., Peter Zwanzger, M.D., Elena Romeo, M.D., Giorgio Bernardi, M.D., Cornelius Schüle, M.D., Thomas C. Baghai, M.D., Robin Ella, M.D., Augusto Pasini, M.D., and Rainer Rupprecht, M.D.

There is evidence for altered levels of neuroactive steroids in major depression that normalize after successful antidepressant pharmacotherapy. Currently it is not known whether this is a general principle of clinically effective antidepressant therapy or a pharmacological effect of antidepressants. Here, we investigated whether repetitive transcranial magnetic stimulation (rTMS) may affect plasma concentrations of neuroactive steroids in a similar way as antidepressant pharmacotherapy. Progesterone, $3 \alpha, 5 \alpha$-tetrahydroprogesterone $(3 \alpha, 5 \alpha-T H P), 3 \alpha, 5 \beta$ tetrahydroprogesterone $(3 \alpha, 5 \beta-T H P), 3 \beta, 5 \alpha-$ tetrahydroprogesterone $(3 \beta, 5 \alpha-T H P)$ and dehydroepiandrosterone (DHEA) were quantified in 37 medication-free patients suffering from a major depressive episode before and after 10 sessions of left prefrontal rTMS.
Plasma samples were analyzed by means of a highly sensitive and specific combined gas chromatography/mass spectrometry analysis. There was a significant reduction of depressive symptoms after $r$ TMS. However, plasma concentrations of neuroactive steroids were not affected by rTMS and not related to clinical response. Clinical improvement after extended daily treatment with rTMS is not accompanied by changes in neuroactive steroid levels. Changes in neuroactive steroid levels after antidepressant pharmacotherapy more likely reflect specific pharmacological effects of antidepressant drugs and are not necessary for the amelioration of depressive symptoms.

[Neuropsychopharmacology 27:874-878, 2002] (C) 2002 American College of Neuropsychopharmacology. Published by Elsevier Science Inc.
From the Department of Psychiatry, Ludwig-Maximilian University, Nussbaumstr. 7, D-80336 Munich, Germany (FP, PZ, CS, TCB, RE, RR), IRCCS Santa Lucia (FdM, ER, GB), and Tor Vergata University (ER, GB, AP), Rome, Italy, and Max Planck Institute of Psychiatry, Kraepelinstr. 2-10, D-89804 Munich, Germany (RR).

Address correspondence to: Rainer Rupprecht, M.D., Department of Psychiatry, Ludwig-Maximilian University, Nussbaumstr.7, 80336 Munich, Germany. Tel.: 01149-89-5160-2770; Fax: 01149-895160-5524; E-mail: Rainer.Rupprecht@psy.med.uni-muenchen.de

Received February 12, 2002; revised April 23, 2002; accepted April 24, 2002

Online publication: $4 / 25 / 02$ at www.acnp.org/citations/ Npp042502294.
KEY WORDS: Affective disorders; Antidepressant treatment; Neuroactive steroids; Prefrontal cortex; Transcranial magnetic stimulation; TMS

Extended treatment with repetitive transcranial magnetic stimulation (rTMS) has been demonstrated to ameliorate symptoms in major depression throughout the majority of controlled clinical trials (Klein et al. 1999; Berman et al. 2000; George et al. 2000; Padberg et al. 2002b). Moreover, several lines of evidence based on behavioral animal models as well as neurochemical findings support the notion that rTMS exerts an antidepressant-like 
action (Fleischmann et al. 1995; Zyss et al. 1997; Ben-Shachar et al. 1999; Kole et al. 1999; Keck et al. 2000).

Neuroactive steroids interacting with the $\gamma$-aminobutyric acid type $\mathrm{A}\left(\mathrm{GABA}_{\mathrm{A}}\right)$ benzodiazepine receptor complex have been suggested to be involved in the pathophysiology of major depression and the action of antidepressant pharmacotherapy (George et al. 1994; Romeo et al. 1998; Uzunova et al. 1998; Rupprecht and Holsboer 1999; Rupprecht et al. 2001). Lowered levels of $3 \alpha$-reduced neuroactive steroids have been found in plasma and cerebrospinal fluid (CSF) of depressed patients (Romeo et al. 1998; Uzunova et al. 1998), which normalized following successful treatment with fluoxetine (Romeo et al. 1998; Uzunova et al. 1998) and other antidepressant drugs (Romeo et al. 1998). On the other hand, dehydroepiandrosterone (DHEA) has been shown to exert beneficial effects on depressive symptoms in a placebo-controlled study (Wolkowitz et al. 1999). In rodents, $3 \alpha$-reduced neuroactive steroids and dehydroepiandrosterone sulfate (DHEAS) have been found to exert antidepressant-like effects in the forced swim test (Khisti et al. 2000; Urani et al. 2001). In addition, the formation of $3 \alpha$-reduced neuroactive steroids is enhanced by treatment with selective serotonin reuptake inhibitors (SSRI) (Uzunov et al. 1996; Griffin and Mellon 1999). Currently it is not known whether the normalization of altered neuroactive steroid concentrations is a prerequisite for the alleviation of depressive symptoms or due to a specific pharmacological action of antidepressant drugs. In the present study we therefore investigated whether repetitive transcranial magnetic stimulation (rTMS) as a novel non-pharmacological treatment in major depression affects plasma concentrations of neuroactive steroids.

\section{METHODS}

Thirty-seven inpatients were included in an open-label protocol (age: $51.5 \pm 14.8$ years, 23 women, 14 men). Patients met DSM-IV criteria for a major depressive episode. All patients gave their written informed consent for this study after the procedure had been fully explained. The study was approved by the local ethical committee. Prior to the study, antidepressant medication and benzodiazepines were washed out for at least seven days and patients remained medication-free during the entire study.

Repetitive transcranial magnetic stimulation was applied as reported elsewhere (Padberg et al. 2002a). Patients underwent 10 sessions of rTMS $(10 \mathrm{~Hz}, 15$ trains of $10 \mathrm{~s}$ each, $30 \mathrm{~s}$ inter-train interval) of the left dorsolateral prefrontal cortex within two weeks at $100 \%$ stimulation intensity related to the individual motor threshold. Severity of depression was assessed using the 21-item Hamilton Rating Scale for Depression
(HRSD) (Hamilton 1960). Response after rTMS was defined as $\geqslant 50 \%$ reduction of the baseline HRSD score and remission by a HRSD score of $\leqslant 9$.

Plasma samples were obtained at 8:00 A.M. before the first rTMS (day 0 ) and the day after the last rTMS session (day 14). After extraction with ethyl acetate, 4-pregnene3,20-dione (progesterone), $5 \alpha$-pregnan- $3 \alpha$-ol-20-one ( $3 \alpha, 5 \alpha-$ tetrahydroprogesterone; $3 \alpha, 5 \alpha$-THP), $5 \beta$-pregnan- $3 \alpha$-ol20 -one ( $3 \alpha, 5 \beta$-tetrahydroprogesterone; $3 \alpha, 5 \beta$-THP), $5 \alpha$ pregnan-3 $3 \beta$-ol-20-one ( $3 \beta, 5 \alpha$-tetrahydroprogesterone; $3 \beta$, $5 \alpha$-THP) and 5-androsten-3 $\beta$-ol-17-one (dehydroepiandrosterone; DHEA) were quantified using a highly sensitive and specific combined gas chromatography/mass spectrometry analysis as previously described (Strohle et al. 2002). A Finningham Trace gas chromatography/mass spectrometry unit equipped with a capillary column was used to analyze the derivatized steroids in the negative ion chemical ionization mode. The detection limit was approximately $10 \mathrm{fmol}$.

Results are expressed as mean \pm SEM. Two-tailed $t$-tests were used to compare HRSD scores before and after rTMS and responders versus non-responders regarding their demographic variables and HRSD scores at baseline. The treatment effect of rTMS on steroid concentrations was tested about significance using a onefactorial multivariate analysis of variance (MANOVA) with treatment as within-subjects factor. Responders and non-responders were compared before and after rTMS by Wilks' multivariate test applying in each case a onefactorial MANOVA with group as between-subjects factor. Correlation analysis was performed using Pearson's correlation coefficients. The level of significance was set at $p<.05$.

\section{RESULTS}

In the overall patient group HRSD scores were significantly reduced from $24.2 \pm 0.9$ at baseline to $15.9 \pm 1.8$ after rTMS $(\mathrm{t}=4.8 ; \mathrm{df}=36 ; p<.001$; Figure 1$)$. After rTMS treatment, $49 \%$ of patients were responders and $32 \%$ were remitters. Demographic variables and baseline HRSD scores did not differ between responders and non-responders.

Plasma concentrations of neuroactive steroids are shown in Figure 2. There was no change in either neuroactive steroid studied after rTMS compared with baseline. This was the case in the overall patient group and when male and female patients were analyzed separately (data not shown). Moreover, MANOVA revealed no significant group effect between responders and nonresponders at baseline or after rTMS treatment. Similarly, there were no significant differences between remitters and non-remitters (data not shown). No significant correlation was found between percent reduction of HRSD scores after rTMS and baseline levels of 


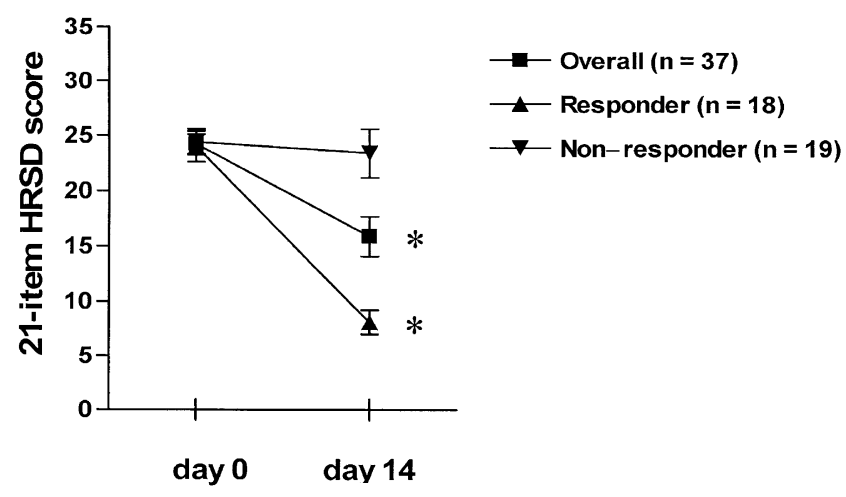

Figure 1. HRSD scores (mean \pm SEM) before (day 0) and after rTMS treatment (day 14) in the overall patient group, in responders and non-responders to rTMS. Significant changes from baseline values are indicated by asterisks ( $p<.01)$.

neuroactive steroids or their changes after treatment $(\mathrm{r}$ ranging from -0.229 to 0.285 ). Baseline HRSD scores and levels of neuroactive steroids at baseline were not correlated either ( $\mathrm{r}$ ranging from -0.196 to 0.267 ).

\section{DISCUSSION}

In contrast to antidepressant pharmacotherapy (Romeo et al. 1998; Uzunova et al. 1998), rTMS did not affect plasma concentrations of neuroactive steroids. There was no change in neuroactive steroid levels associated with clinical recovery in spite of moderate to substantial antidepressant effects of rTMS treatment.

Recently, $3 \alpha$-reduced neuroactive steroids $(3 \alpha, 5 \alpha-$ THP and $3 \alpha, 5 \beta$-THP) have been proposed to play a causal role in the pathogenesis of major depression and its treatment (Romeo et al. 1998; Rupprecht and Holsboer 1999; Rupprecht et al. 2001). $3 \alpha, 5 \alpha$-THP and $3 \alpha, 5 \beta$-THP are potent positive allosteric modulators of various $\mathrm{GABA}_{\mathrm{A}}$-receptor subtypes (Rupprecht et al. 2001).
Moreover, $3 \alpha, 5 \alpha$-THP exerts antidepressant like effects in the forced swim test which can be potentiated by serotonergic agents (Khisti et al. 2000; Khisti and Chopde 2000).

Concentrations of $3 \alpha, 5 \alpha$-THP and $3 \alpha, 5 \beta$-THP are decreased in plasma and cerebrospinal fluid during depression and normalize during treatment with fluoxetine and other antidepressant drugs (Romeo et al. 1998; Uzunova et al. 1998). In contrast, plasma concentrations of $3 \beta, 5 \alpha-\mathrm{THP}$, an antagonistic isomer of GABAergic steroids, are increased in depression (Romeo et al. 1998). In previous studies it has not been possible to differentiate effects due to amelioration of clinical symptoms from direct pharmacological effects of antidepressants. In view of recent molecular data showing that selective serotonin reuptake inhibitors may shift the activity of the $3 \alpha$-hydroxysteroid oxidoreductase toward the reductive direction, thereby increasing the formation of endogenous $3 \alpha$-reduced neuroactive steroids (Griffin and Mellon 1999), it is of particular interest to investigate the action of non-pharmacological antidepressant interventions on the concentrations of neuroactive steroids. Increased concentrations of DHEAS, the sulfate derivative of DHEA, have been suggested to predict non-response to electroconvulsive therapy (ECT) (Maayan et al. 2000). However, concentrations of $3 \alpha$-reduced neuroactive steroids during ECT have not been investigated to date.

In the present study, no changes in neuroactive steroid levels were found after clinical improvement following rTMS treatment. Therefore, the previously reported changes in neuroactive steroid concentrations following antidepressant pharmacotherapy (Romeo et al. 1998; Uzunova et al. 1998) more likely reflect specific pharmacological effects of antidepressant drugs (Griffin and Mellon 1999) that may contribute to symptom improvement, rather than a common mechanism leading to the alleviation of depressive symptoms following successful treatment in general, regardless of the treat-
A

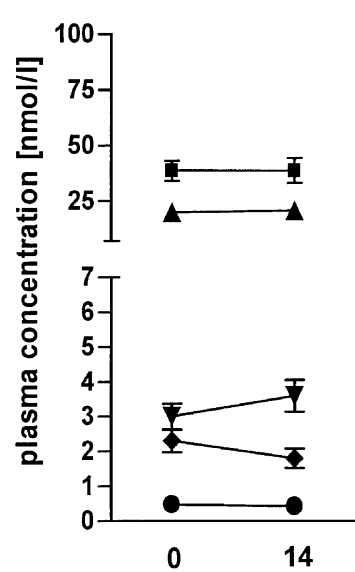

B

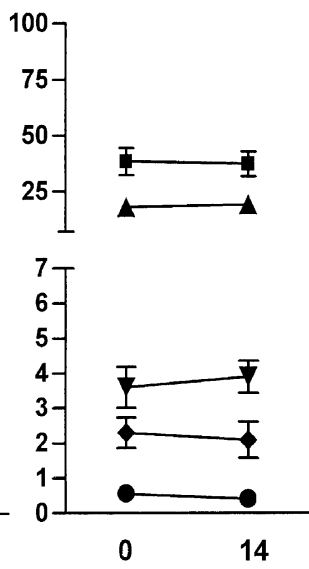

C

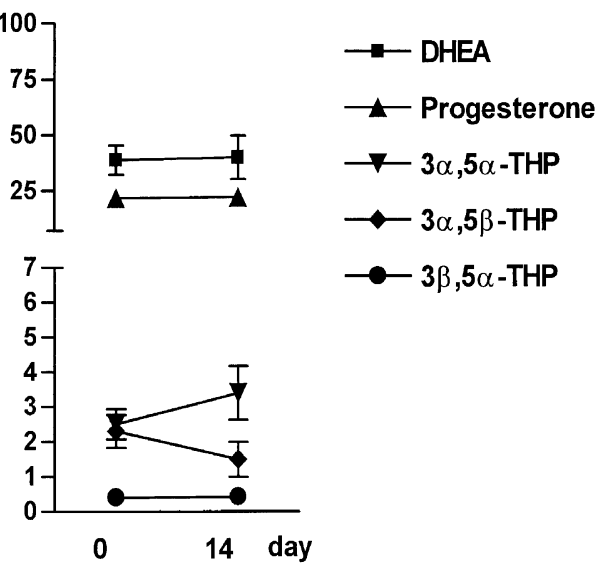

Figure 2. Plasma concentrations of neuroactive steroids (mean \pm SEM) in (A) the overall patient group $(n=37)$, (B) responders $(\mathrm{n}=18)$ and (C) non-responders $(\mathrm{n}=19)$ before (day 0 ) and after rTMS treatment (day 14). The changes in mean $3 \alpha, 5 \alpha$-THP and $3 \alpha, 5 \beta$-THP levels after rTMS treatment did not reach statistical significance. 
ment modality. The open label study design and the fact that rTMS is not yet a clinically established antidepressant intervention are limitations for the interpretation of our findings. Clinical improvement after rTMS may be due to a specific effect of rTMS or a placebo effect, or more likely a combination of both. Nevertheless, changes in neuroactive steroid levels are not mandatory for the alleviation of depressive symptoms. Future studies should investigate the influence of other non-pharmacological treatments of depression, e.g. sleep deprivation, $\mathrm{ECT}$, or vagus nerve stimulation (VNS), on the concentrations of neuroactive steroids. Although changes in neuroactive steroids apparently do not explain the antidepressant efficacy of rTMS, these molecules nevertheless may contribute to the efficacy of treatment with antidepressant drugs in various psychiatric disorders.

\section{ACKNOWLEDGMENTS}

The authors wish to thank Dr. Patrick Mikhaiel and Dr. Heike Thoma for conducting the rTMS treatment. They further thank Mr. J. Scandone and Mr. Peter Houseman from the Magstim Company for their technical support. This project was supported by the German Ministry for Education and Research within the promotional emphasis "German Research Network on Depression" (Subproject 4.3, R.R., Subproject 6.5, F.P.), the Gerhard Hess Program of the German Research Foundation and the Max-Planck Society.

\section{REFERENCES}

Ben-Shachar D, Gazawi H, Riboyad-Levin J, Klein E (1999): Chronic repetitive transcranial magnetic stimulation alters beta-adrenergic and 5-HT2 receptor characteristics in rat brain. Brain Res 816:78-83

Berman RM, Narasimhan M, Sanacora G, Miano AP, Hoffman RE, Hu XS, Charney DS, Boutros NN (2000): A randomized clinical trial of repetitive transcranial magnetic stimulation in the treatment of major depression. Biol Psychiatry 47:332-337

Fleischmann A, Prolov K, Abarbanel J, Belmaker RH (1995): The effect of transcranial magnetic stimulation of rat brain on behavioral models of depression. Brain Res 699:130-132

George MS, Guidotti A, Rubinow D, Pan B, Mikalauskas K, Post RM (1994): CSF neuroactive steroids in affective disorders: pregnenolone, progesterone and DBI. Biol Psychiatry 35:775-780

George MS, Nahas Z, Molloy M, Speer AM, Oliver NC, Li X-B, Arana GW, Risch SC, Ballenger JC (2000): A controlled trial of daily left prefrontal cortex TMS for treating depression. Biol Psychiatry 48:962-970

Griffin LD, Mellon SH (1999): Selective serotonin reuptake inhibitors directly alter activity of neurosteroidogenic enzymes. Proc Natl Acad Sci USA 96:13512-13517

Hamilton M (1960): A rating scale for depression. J Neurol Neurosurg Psychiatry 23:56-62
Keck ME, Engelmann M, Müller MB, Henniger MSH, Hermann B, Rupprecht R, Neumann ID, Toschi N, Landgraf R, Post A (2000): Repetitive transcranial magnetic stimulation induces active coping strategies and attenuates the neuroendocrine stress response in rats. J Psychiatr Res 34:265-276

Khisti RT, Chopde CT, Jain SP (2000): Antidepressant-like effect of the neurosteroid $3 \alpha$-hydroxy- $5 \alpha$-pregnan-20one in mice forced swim test. Pharmacol Biochem Behav 67:137-143

Khisti RT, Chopde CT (2000): Serotonergic agents modulate antidepressant-like effect of the neurosteroid $3 \alpha$-hydroxy5 $\alpha$-pregnan-20-one in mice. Brain Res 865:291-300

Klein E, Kreinin I, Chistyakov A, Koren D, Mecz L, Marmur S, Ben-Shachar D, Feinsod M (1999): Therapeutic efficacy of right prefrontal slow repetitive transcranial magnetic stimulation in major depression. Arch Gen Psychiatry 56:315-320

Kole MH, Fuchs E, Ziemann U, Paulus W, Ebert U (1999): Changes in $5-\mathrm{HT}_{1 \mathrm{~A}}$ and NMDA binding sites by a single rapid transcranial magnetic stimulation procedure in rats. Brain Res 826:309-312

Maayan R, Yagorowski Y, Grupper D, Weiss M, Shtaif B, Kaoud MA, Weizman A (2000): Basal plasma dehydroepiandrosterone sulfate level: a possible predictor for response to electroconvulsive therapy in depressed psychotic inpatients. Biol Psychiatry 48:693-701

Padberg F, Schüle C, Zwanzger P, Baghai T, Ella R, Mikhaiel P, Hampel H, Möller HJ, Rupprecht R (2002a): Relation between responses to repetitive transcranial magnetic stimulation and partial sleep deprivation in major depression. J Psychiatr Res 36:131-135

Padberg F, Zwanzger P, Keck ME, Kathmann N, Mikhaiel P, Ella R, Rupprecht P, Thoma H, Hampel H, Toschi N, Möller HJ (2002b): Repetitive transcranial magnetic stimulation (rTMS) in major depression: relation between efficacy and stimulation intensity. Neuropsychopharmacology (in press)

Romeo E, Strohle A, Spalletta G, di Michele F, Hermann B, Holsboer F, Pasini A, Rupprecht R (1998): Effects of antidepressant treatment on neuroactive steroids in major depression. Am J Psychiatry 155:910-913

Rupprecht R, di Michele F, Hermann B, Strohle A, Lancel M, Romeo E, Holsboer F (2001): Neuroactive steroids: molecular mechanisms of action and implications for neuropsychopharmacology. Brain Res Rev 37:59-67

Rupprecht R, Holsboer F (1999): Neuroactive steroids: mechanisms of action and neuropsychopharmacological perspectives. Trends Neurosci 22:410-416

Strohle A, Romeo E, di Michele F, Pasini A, Yassouridis A, Holsboer F, Rupprecht R (2002): GABA(A) receptormodulating neuroactive steroid composition in patients with panic disorder before and during paroxetine treatment. Am J Psychiatry 159:145-147

Urani A, Roman FJ, Phan VL, Su TP, Maurice T (2001): The antidepressant-like effect induced by sigma(1)-receptor agonists and neuroactive steroids in mice submitted to the forced swimming test. J Pharmacol Exp Ther 298: 1269-1279

Uzunov DP, Cooper TB, Costa E, Guidotti A (1996): Fluoxetine-elicited changes in brain neurosteroid content measured by negative ion mass fragmentography. Proc Natl Acad Sci USA 93:12599-12604 
Uzunova V, Sheline Y, Davis JM, Rasmusson A, Uzunov DP, Costa E, Guidotti A (1998): Increase in the cerebrospinal fluid content of neurosteroids in patients with unipolar major depression who are receiving fluoxetine or fluvoxamine. Proc Natl Acad Sci USA 95:3239-3244

Wolkowitz OM, Reus VI, Keebler A, Nelson N, Friedland M, Brizendine L, Roberts E (1999): Double-blind treatment of major depression with dehydroepiandrosterone. Am J Psychiatry 156:646-649

Zyss T, Gorka Z, Kowalska M, Vetulani J (1997): Preliminary comparison of behavioral and biochemical effects of chronic transcranial magnetic stimulation and electroconvulsive shock in the rat. Biol Psychiatry 42:920924 